\title{
Association of vancomycin trough concentration on the treatment outcome of patients with bacteremia caused by Enterococcus species
}

Yujin Sohn ${ }^{1,2+}$, John Hoon Rim ${ }^{3 \dagger}$, Yunsuk Cho ${ }^{1,2}$, Jonghoon Hyun ${ }^{1,2}$, Yaejee Baek ${ }^{1,2}$, Moohyun Kim ${ }^{1,2}$, Jung Ho Kim ${ }^{1,2}$, Hye Seong ${ }^{1,2}$, Jin Young Ahn 1,2, Sang-Guk Lee ${ }^{3^{*}}$, Jong-Beack Lim³ ${ }^{3}$, Su Jin Jeong ${ }^{1,2}$, Nam Su Ku ${ }^{1,2^{*}}$, Jun Yong Choi ${ }^{1,2}$, Joon-Sup Yeom ${ }^{1,2}$ and Young Goo Song ${ }^{1,2}$

\begin{abstract}
Background: Pharmacokinetic-pharmacodynamic (PK/PD) targets of vancomycin therapy have been recognized for methicillin-resistant Staphylococcus aureus infections but not for other gram-positive bacterial infections. Therefore, we investigated whether vancomycin concentration targets such as the trough level and ratio of the area under the curve to minimum inhibitory concentration (AUC/MIC) are associated with the treatment outcome in enterococcal bacteremia.

Methods: A retrospective cohort analysis enrolled patients with bacteremia caused by vancomycin-susceptible Enterococcus faecium and Enterococcus faecalis who were treated with vancomycin from January 2007 to December 2017 at a tertiary hospital located in Seoul, South Korea. Patients without vancomycin concentrations were excluded from the study. The primary outcome was 28-day all-cause mortality.

Results: A total of 37 patients were enrolled-26 with E. faecium infection and 11 with E. faecalis infection. The 28 -day all-cause mortality rate was $21.6 \%$. In univariate analysis, vancomycin trough level $(\leq 15 \mu \mathrm{g} / \mathrm{mL} ; p=0.042)$, age $(p=0.044)$, and septic shock $(p=0.049)$ were associated with 28-day mortality but not AUC24/MIC (> 389; $p$ $=0.479)$. In multivariate analysis, vancomycin trough concentration $(\leq 15 \mu \mathrm{g} / \mathrm{mL} ; p=0.041)$ and younger age $(p=$ 0.031 ) were associated with 28-day mortality in patients with enterococcal bacteremia.
\end{abstract}

Conclusions: In this study, a vancomycin trough level of $15 \mu \mathrm{g} / \mathrm{mL}$ or lower was associated with 28-day mortality in enterococcal bacteremia. However, relatively large prospective studies are needed to examine the efficacy of vancomycin PK/PD parameters in patients with enterococcal bacteremia.

Keywords: Vancomycin, Enterococcus, Trough level, AUC/MIC

*Correspondence: comforter6@yuhs.ac; smileboy9@yuhs.ac

${ }^{\dagger}$ Yujin Sohn and John Hoon Rim contributed equally to this study 1 Department of Internal Medicine, Yonsei University College of Medicine,

50-1 Yonsei-ro, Seodaemun-gu, Seoul 03722, Republic of Korea

${ }^{3}$ Department of Laboratory Medicine, Yonsei University College

of Medicine, 50-1 Yonsei-ro, Seodaemun-gu, Seoul 03722, Republic

of Korea

Full list of author information is available at the end of the article

\section{Background}

Enterococcus species are ubiquitous in natural environments, including plants, soil, and water, and are part of the normal gastrointestinal flora of humans and other animals. Their broad distribution allows them to survive and persist in various environments [1]. Because of their ability to colonize medical devices and their high viability 
in nosocomial environments, Enterococcus species are a major cause of hospital-acquired infections [2]. Enterococci are important nosocomial pathogens worldwide, accounting for $14 \%$ of all hospital-acquired infections in the United States between 2011 and 2014 [3]. Enterococcus species cause infections of the bloodstream, urinary tract, and surgical sites, inter alia. These infections can lead to infective endocarditis, urinary tract infections, bacteremia, peritonitis, and prosthetic joint infections. In many centers, the proportion of ampicillin-resistant Enterococcus faecium exceeds 70\%. Enterococcus faecalis resistant to ampicillin is rare, but resistance rate is increasing in nosocomial infections and the rate of ampicillin-resistant E. faecalis is reported to be $1.8 \%$; hence, the use of vancomycin is increasing [3, 4].

Vancomycin is the commonly used glycopeptide to treat aminopenicillin-resistant gram-positive bacteria [5]. Because it has adverse effects such as nephrotoxicity, studies have examined the pharmacokinetics of vancomycin to minimize its toxicity and maximize its therapeutic effect [6]. The guidelines for vancomycin dosing strategies to assist clinicians and pharmacists have been revised. By attempting to implement therapeutic monitoring using current knowledge of vancomycin pharmacodynamics and in view of potential problems with efficacy, resistance, and toxicity, these guidelines are intended to improve patient outcomes [7, 8]. Vancomycin is the drug of choice for methicillin-resistant Staphylococcus aureus (MRSA) infections $[8,9]$. The pharmacodynamic parameter that best predicts vancomycin efficacy in invasive MRSA infections is the ratio of the $24 \mathrm{~h}$ area under the concentration-time curve to minimum inhibitory concentration $\left(\mathrm{AUC}_{24} / \mathrm{MIC}\right)$ [10]. Serum vancomycin trough concentrations have been used as a surrogate marker for identifying an $\mathrm{AUC}_{24} / \mathrm{MIC}$ of $\geq 400$ [11], although several recent studies found that the trough concentration poorly reflects the $\mathrm{AUC}_{24} / \mathrm{MIC}$ target and that a high trough concentration increases the risk of nephrotoxicity $[8,12]$.

To our knowledge, few studies have examined the target vancomycin concentration in the treatment of nonMRSA gram-positive infections, especially those caused by Enterococcus species. Therefore, we investigated whether vancomycin concentration targets such as the trough level and AUC/MIC are associated with the treatment outcome in patients with bacteremia caused by Enterococcus species.

\section{Methods}

\section{Study population and design}

A retrospective, single-center, cohort study was conducted at Severance Hospital, a 2400-bed, tertiary-care hospital in Seoul, South Korea. The electronic medical records of patients $>18$ years of age with enterococcal bacteremia treated with vancomycin between January 1, 2007 and December 31, 2017 were reviewed. The age range of patients enrolled in this study was from 26 to 91 years. The exclusion criteria were as follows: presence of polymicrobial bacteremia, isolates resistant to vancomycin, missing trough vancomycin concentration, and vancomycin MIC data. Data collected from the patients' medical records included demographic characteristics, comorbidities, source of bacteremia, antimicrobial treatment data, duration of bacteremia, relapse of bacteremia, and mortality. The primary outcome was 28-day all-cause mortality. The study was approved by the Institutional Review Board (IRB) of the Yonsei University Health System Clinical Trial Center (4-2020-0161). All methods were carried out in accordance with relevant guidelines and regulations under Ethics approval. Since the study was retrospective and the study subjects were anonymized, the IRB waived the requirement for written informed consent from the patients.

\section{Definitions}

Bacteremia was defined as at least one positive blood culture with an identifiable source and clinical manifestations consistent with bacteremia, or at least two separate blood cultures positive for Enterococcus species. Persistent blood stream infection was defined as the case when the bacteria were continuously identified in two or more follow-up cultures after the first positive Enterococcus blood culture [13]. For patients with more than one episode of Enterococcus bacteremia, only the first episode during the study period was included. The duration of bacteremia was defined as the number of days from the first positive blood culture to the date of the first negative Enterococcus blood culture. Relapse occurred if the cultures became negative for more than 2 days and then became positive within 90 days. Vancomycin-induced nephropathy (VIN) was defined as an increase in the serum creatinine level of $\geq 0.5 \mathrm{mg} / \mathrm{dL}$, or a $50 \%$ increase from baseline in consecutive daily readings, or a decrease in the calculated creatinine level of $50 \%$ from baseline on 2 consecutive days in the absence of an alternative explanation [6]. The inappropriate empirical antibiotics use means that the empirical antibiotics used before identification of the bacteria is not suitable for the drug susceptibility test result. Community-acquired bacteremia is defined as bacteremia occurred within $48 \mathrm{~h}$ of hospitalization. And hospital-acquired bacteremia is a bacteremia occurred $48 \mathrm{~h}$ after admission.

\section{Microbiological data}

The species in the clinical isolates were identified using the VITEK ${ }^{\circledR} 2$ system with GNI cards (bioMérieux, 
Marcy-l'Étoile, France) until 2014 or Microflex MALDITOF mass spectrometry with Biotyper software 3.1 (Bruker Daltonics, Leipzig, Germany) after clinical adoption in 2014. Antimicrobial susceptibility tests were performed using the disk diffusion method or a VITEK-2 N131 card (bioMérieux). The results were interpreted according to the Clinical and Laboratory Standards Institute guidelines [14].

\section{Vancomycin dosing and pharmacodynamics data}

To obtain trough concentrations, blood samples were collected immediately before the next vancomycin dose. Samples for peak concentrations were collected $1 \mathrm{~h}$ after intravenous vancomycin infusion was completed. Based on the hospital guidelines, all venous blood samples used to determine steady-state serum concentrations were obtained after administering at least four doses of vancomycin. The serum vancomycin concentrations were analyzed with a chemiluminescence microparticle immunoassay using an Architect automated immunochemistry analyzer (Abbott Labs, Chicago, IL, USA). All pharmacokinetic calculations and modeling were performed using the MW/Pharm software package (ver. 3.82; Mediware, Zuidhorn, the Netherlands). And the trough level used to categorize in this study was measured through the first blood samples after at least four consecutive doses of vancomycin.

Demographic parameters, including weight, height, ethnicity, and sex, were imported from the hospital's electronic records. Renal function was estimated using the most recent serum creatinine level. Estimated curves were fitted using the default settings of the posterior Bayesian estimation after the simulation. The area under the curve (AUC) of the estimated vancomycin concentration was calculated in a chronological manner. Patients on renal dialysis were screened for additional creatinine concentrations.

\section{Statistical analysis}

The relationships between the vancomycin trough concentration or the $\mathrm{AUC}_{24} / \mathrm{MIC}$ and mortality in patients with E. faecium or E. faecalis bacteremia were analyzed using both continuous and categorical variables. The Kolmogorov-Smirnov test and Shapiro-Wilk test were conducted to verify the normality of the continuous variables. The independent $t$-test and the Mann-Whitney test were used to compare the continuous variables of the two groups. The chi-square test or Fisher's exact test were used to compare categorical variables. Potentially significant variables identified in the univariate analysis were included in a multivariate logistic regression analysis to identify the risk factors associated with 28-day mortality. The Kaplan-Meier survival curve was used to compare mortality according to vancomycin trough concentrations. All statistical analyses were performed using SPSS Statistics ver. 25.0 (IBM Corp., Armonk, NY, USA).

\section{Results}

A total of 209 patients had confirmed enterococcal bacteremia. Of these, 73 patients had polymicrobial bacteremia, 62 had vancomycin-resistant enterococci, and 37 did not perform therapeutic drug monitoring (TDM). Finally, 37 patients ( 22 men, 15 women; mean age, 60.4 years) were enrolled: 26 cases of E. faecium and 11 of $E$. faecalis. (Additional file 1: Table S1). The most common comorbidities were solid cancer $(56.8 \%)$, followed by hypertension (40.5\%), and diabetes mellitus (35.1\%). The most common source of infection was biliary infection (40.5\%), followed by peritonitis (21.6\%).

The 28 -day mortality rate was $21.6 \%(8 / 37)$. No significant differences were found in the baseline characteristics, except for age (Table 1). Vancomycin-induced nephropathy occurred in 6 of the 37 patients. In the MIC distributions for vancomycin, there were 3 cases of MIC $2 \mathrm{mg} / \mathrm{L}, 18$ cases of MIC $1 \mathrm{mg} / \mathrm{L}$, and 16 cases of MIC < $0.5 \mathrm{mg} / \mathrm{L}$. The median value of $\mathrm{AUC}_{24}$ in this study was 474.21, the minimum value was 181.17 , and the maximum was 1111.41.

In univariate analyses, vancomycin trough concentration $(\leq 15 \mu \mathrm{g} / \mathrm{mL})(p=0.042)$, age $(p=0.044)$, and septic shock $(p=0.049)$ were associated with 28 -day mortality. The proportion of patients achieving $\mathrm{AUC}_{24} / \mathrm{MIC} \leq$ 389 did not differ between the two groups $(p=0.479)$. In a multivariate analysis, vancomycin trough concentration $(\leq 15 \mu \mathrm{g} / \mathrm{mL})(p=0.041$; odds ratio (OR), 119.013; 95\% confidence interval (CI), [1.207-11732.690]), and younger age $(p=0.031$; OR, 1.212 ; 95\% CI, [1.0181.442]) were associated with mortality in patients with enterococcal bacteremia (Table 2). Moreover, in the Kaplan-Meier analysis, the group with vancomycin trough levels of $15 \mu \mathrm{g} / \mathrm{mL}$ or lower had a higher 28-day mortality $(\log$ rank $=0.027)$ (Fig. 1$)$.

\section{Discussion}

In this study, we found that vancomycin trough concentration $(\leq 15 \mu \mathrm{g} / \mathrm{mL})$ was significantly associated with increased mortality in patients with Enterococcus bacteremia.

Many studies have examined pharmacokinetic/pharmacodynamic (PK/PD) parameters to maintain the efficiency of vancomycin and to minimize its adverse effects, but most of these studies have examined MRSA infection. Of the PK/PD parameters, the trough level and $\mathrm{AUC}_{24} /$ MIC have been reported to describe the effectiveness of vancomycin $[15,16]$. The clinical success and renal toxicity of vancomycin treatment are exposure-dependent and 
Table 1 Baseline demographic and clinical features of patients with enterococcal bacteremia

\begin{tabular}{|c|c|c|c|c|}
\hline \multirow[b]{2}{*}{ Characteristics } & \multirow[b]{2}{*}{ Total $(n=37)$} & \multicolumn{2}{|c|}{ 28-day all mortality } & \multirow[b]{2}{*}{$P$ value } \\
\hline & & Survivors $(n=29)$ & Non-survivors $(n=8)$ & \\
\hline \multicolumn{5}{|l|}{ Demographics } \\
\hline Age (year, mean $\pm S D$ ) & $60.5 \pm 13.2$ & $62.8 \pm 2.3$ & $52.3 \pm 4.4$ & 0.044 \\
\hline $\mathrm{BMI}($ mean $\pm \mathrm{SD})$ & $21.4 \pm 3.1$ & $21.2 \pm 0.6$ & $22.4 \pm 1.1$ & 0.323 \\
\hline Male (\%) & $22(59.5)$ & $18(62.1)$ & $4(50.0)$ & 0.412 \\
\hline Community $\mathrm{AB}(\%)$ & $9(24.3)$ & $7(24.1)$ & $2(25.0)$ & 0.643 \\
\hline Hospital AB (\%) & $28(75.7)$ & $22(75.9)$ & $6(75.0)$ & 0.643 \\
\hline \multicolumn{5}{|l|}{ Comorbidities (\%) } \\
\hline Solid cancer & $21(56.8)$ & $17(58.6)$ & $4(50.0)$ & 0.483 \\
\hline HTN & $15(40.5)$ & $11(37.9)$ & $4(50.0)$ & 0.412 \\
\hline DM & $13(35.1)$ & $11(37.9)$ & $2(25.0)$ & 0.408 \\
\hline Chronic liver disease & $11(29.7)$ & $8(27.6)$ & $3(37.5)$ & 0.444 \\
\hline Organ transplantation & $9(24.3)$ & $7(24.1)$ & $2(25.0)$ & 0.643 \\
\hline Chronic renal disease & $9(24.3)$ & $6(20.7)$ & $3(37.5)$ & 0.292 \\
\hline Cerebrovascular disease & $7(18.9)$ & $6(20.7)$ & $1(12.5)$ & 0.521 \\
\hline Cardiovascular disease & $6(16.2)$ & $5(17.2)$ & $1(12.5)$ & 0.613 \\
\hline Hematologic malignancies & $4(10.8)$ & $3(10.3)$ & $1(12.5)$ & 0.640 \\
\hline $\mathrm{CHF}$ & $3(8.1)$ & $2(6.9)$ & $1(12.5)$ & 0.530 \\
\hline Hemiplegia & $2(5.4)$ & $2(6.9)$ & $0(0.0)$ & 0.610 \\
\hline PAOD & $1(2.7)$ & $0(0.0)$ & $1(12.5)$ & 0.216 \\
\hline ILD & $1(2.7)$ & $1(3.4)$ & $0(0.0)$ & 0.784 \\
\hline Antibiotic use in 30 day (\%) & $27(73.0)$ & $22(75.9)$ & $5(62.5)$ & 0.367 \\
\hline Steroid use in 30 day (\%) & $16(43.2)$ & $12(41.4)$ & $4(50.0)$ & 0.483 \\
\hline Anticancer drug use in 30 day (\%) & $9(24.3)$ & $7(24.1)$ & $2(25.0)$ & 0.643 \\
\hline Immunosuppressant use in 30 day (\%) & $9(24.3)$ & $7(24.1)$ & $2(25.0)$ & 0.643 \\
\hline Vancomycin induced AKI (\%) & $6(16.2)$ & $4(13.8)$ & $2(25.0)$ & 0.591 \\
\hline Septic shock (\%) & $8(21.6)$ & $4(13.8)$ & $4(50.0)$ & 0.049 \\
\hline \multicolumn{5}{|l|}{ Source of bacteremia (\%) } \\
\hline Biliary & $15(40.5)$ & $12(41.4)$ & $3(37.5)$ & 0.588 \\
\hline Peritonitis & $8(21.6)$ & $7(24.1)$ & $1(12.5)$ & 0.435 \\
\hline UTI & $1(2.7)$ & $0(0.0)$ & $1(12.5)$ & 0.216 \\
\hline Skin & $1(2.7)$ & $1(3.4)$ & $0(0.0)$ & 0.784 \\
\hline CRBSI & $1(2.7)$ & $1(3.4)$ & $0(0.0)$ & 0.784 \\
\hline Foreign device & $1(2.7)$ & $1(3.4)$ & $0(0.0)$ & 0.784 \\
\hline Others & $2(5.4)$ & $1(3.4)$ & $1(12.5)$ & 0.390 \\
\hline Primary & $8(21.6)$ & $6(20.7)$ & $2(25.0)$ & 0.565 \\
\hline ICU stay (\%) & $14(37.8)$ & $10(34.5)$ & $4(50.0)$ & 0.343 \\
\hline SOFA score (mean \pm SD) & $7.0 \pm 4.8$ & $6.2 \pm 0.8$ & $9.9 \pm 1.9$ & 0.056 \\
\hline Persistent BSI (\%) & 18 (48.6) & $15(51.7)$ & $3(37.5)$ & 0.379 \\
\hline Recurrence of same BSI (\%) & $5(13.5)$ & $5(17.2)$ & $0(0.0)$ & 0.272 \\
\hline Initial empirical inappropriate antibiotics (\%) & $16(43.2)$ & $13(44.8)$ & $3(37.5)$ & 0.517 \\
\hline Combination therapy (\%) & $8(21.6)$ & $6(20.7)$ & $2(25.0)$ & 0.565 \\
\hline \multicolumn{5}{|l|}{ PK/PD parameter $(\%)$} \\
\hline Trough level $\leq 15 \mu \mathrm{g} / \mathrm{mL}$ & $19(51.4)$ & $12(41.4)$ & $7(87.5)$ & \\
\hline Trough level $>15 \mu \mathrm{g} / \mathrm{mL}$ & 18 (48.6) & $17(58.6)$ & $1(12.5)$ & 0.042 \\
\hline $\mathrm{AUC}_{24} / \mathrm{MIC} \leq 389$ & $7(18.9)$ & $5(17.2)$ & $2(25.0)$ & \\
\hline $\mathrm{AUC}_{24} / \mathrm{MIC}>389$ & $30(81.1)$ & $24(82.8)$ & $6(75.0)$ & 0.479 \\
\hline
\end{tabular}


Table 1 (continued)

\section{8-day all mortality}

BMI, body mass index; Community AB, Community acquired bacteremia; Hospital AB, Hospital acquired bacteremia; HTN, hypertension; DM, diabetes mellitus; CHF, congestive heart failure; PAOD peripheral arterial occlusive disease; ILD, interstitial lung disease; AKI. Acute kidney disease, UTI, urinary tract infection; CRBSI, catheter-related blood stream infection; BSI, blood stream infection, AUC, area under curve; MIC minimum inhibitory concentration; Other cases of bacteremia include infective endocarditis

Continuous variables are shown as the mean \pm standard deviation (SD) and categorical variables, as numbers (percentage)

Table 2 Multivariate logistic regression analysis for factors associated with 28-day mortality

\begin{tabular}{llll}
\hline Variables & OR & $\mathbf{9 5 \% ~ C l}$ & $\boldsymbol{p}$ value \\
\hline Younger age & 1.212 & $1.018 \sim 1.442$ & 0.031 \\
$\begin{array}{l}\text { Vancomycin trough } \\
\text { level } \leq 15 \mu \mathrm{g} / \mathrm{mL}\end{array}$ & 119.013 & $1.207 \sim 11732.690$ & 0.041 \\
Septic shock & 18.369 & $0.997 \sim 338.574$ & 0.050 \\
\hline
\end{tabular}

$\mathrm{OR}$, odds ratio; $\mathrm{Cl}$, confidence interval

characterized by the $\mathrm{AUC}_{24}$. The exact range targeted by clinicians is influenced by the $\mathrm{AUC}_{24}$ estimation and bacterial MIC $[15,17]$. Using AUC-based vancomycin therapeutic drug monitoring (TDM) helps to individualize the estimation of the $\mathrm{AUC}_{24} / \mathrm{MIC}$ and minimize the risk of toxicity due to unnecessary overexposure.

Although trough concentration is a known surrogate marker for $\mathrm{AUC}_{24} / \mathrm{MIC}$, the method using only troughbased vancomycin TDM is controversial for several reasons. First, the vancomycin trough concentration poorly characterizes the $\mathrm{AUC}_{24}$, and adequate vancomycin $\mathrm{AUC}_{24}$ levels can be obtained at trough concentrations $<15 \mathrm{mg} / \mathrm{L}$. Vancomycin-associated nephrotoxicity also increases when the vancomycin trough concentration is $>15 \mathrm{mg} / \mathrm{L}$. These factors provide evidence for an AUC-based approach to vancomycin TDM [18]. Thus, according to the Infectious Diseases Society of America guidelines, the trough level does not correlate well with the AUC, and trough-only monitoring with a target of $15-20 \mu \mathrm{g} / \mathrm{mL}$ is no longer recommended based on its efficacy in patients with MRSA infections [10]. However, because it may be difficult to determine the $\mathrm{AUC}_{24} / \mathrm{MIC}$ in a clinical setting, trough serum concentrations are still monitored clinically. Moreover, there is insufficient evidence to recommend whether trough level-only or AUC/ MIC-guided vancomycin monitoring should be used for patients with non-MRSA infections.

In one study of PK/PD determinants of vancomycin efficacy in enterococcal bacteremia, a vancomycin AUC/MIC $\geq 389$ achieved within $72 \mathrm{~h}$ was associated with reduced mortality. However, the study found that

Kaplan-Meier Curves

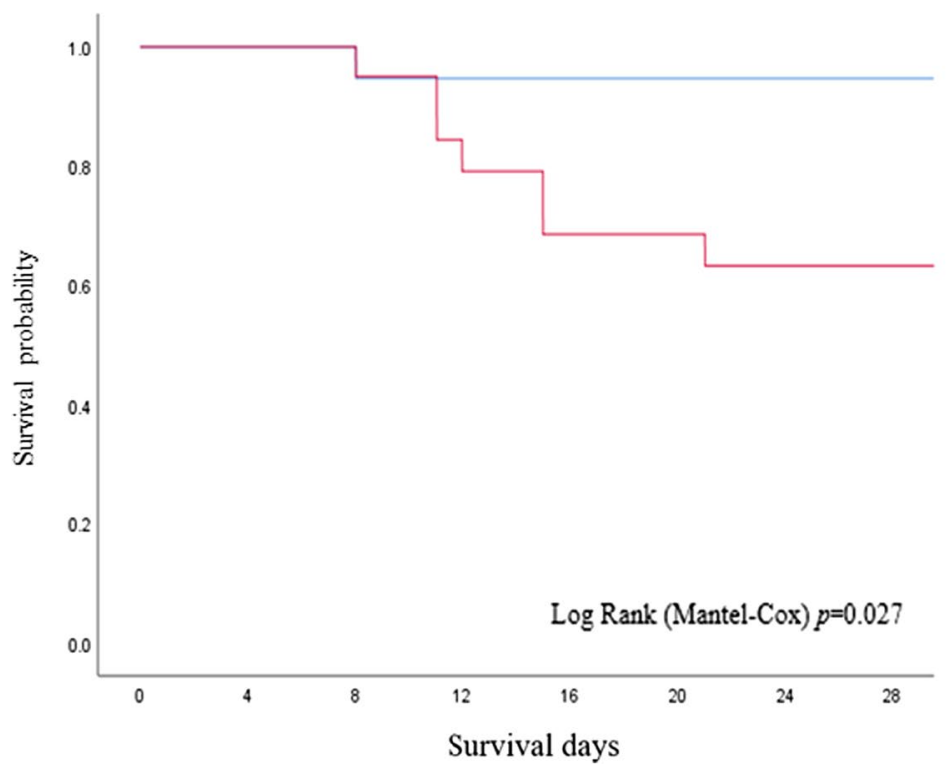

Fig. 1 Kaplan-Meier survival analysis of patients according to vancomycin trough level 
vancomycin trough concentrations differed significantly between survivors and non-survivors [19]. According to Nakamura et al. [20] neither the $\mathrm{AUC}_{24} / \mathrm{MIC}$ nor the trough concentration of vancomycin is significantly associated with mortality in patients with E. faecium bacteremia. The trough concentration was higher in nonsurvivors than in survivors, and $\mathrm{AUC}_{24} / \mathrm{MIC}$ did not differ significantly between non-survivors and survivors.

In our study, vancomycin trough levels of $15 \mu \mathrm{g} / \mathrm{mL}$ or lower were associated with mortality in patients with enterococcal bacteremia, but the $\mathrm{AUC}_{24} / \mathrm{MIC}$ was not. Zelenitsky et al. reported that the survival rate was higher when the vancomycin trough concentration was maintained above $15 \mathrm{mg} / \mathrm{L}$ than when low trough concentrations were maintained [21]. In addition, Kullar et al. reported that the vancomycin treatment period was significantly shorter in the group in which the vancomycin trough concentration was higher than the group to which the vancomycin trough concentration was applied at 5 to $20 \mathrm{mg} / \mathrm{L}$, and the rate at which the bacteria were negatively converted was also found to be significantly higher in the group that maintained the higher trough concentration [22]. According to a meta-analysis comparing high and low vancomycin serum trough regimen groups with gram-positive bacterial infections, there was no significant difference between the two in all-cause mortality or risk of clinical failure; however, a subgroup analysis confirmed that treatment failure was reduced in high vancomycin trough regimen groups [23]. Therefore, in enterococcal bacteremia, the trough level may be a good parameter for monitoring the therapeutic effects of vancomycin.

All enterococcal BSIs treated with vancomycin were included in this study to examine the therapeutic effect of vancomycin on Enterococcus species. Therefore, strains susceptible to ampicillin were added in this study. In most cases, vancomycin was used for ampicillin-susceptible Enterococcus species when beta-lactam antibiotics could not be used due to its side effects, or was used without checking the results of antimicrobial susceptibility tests. Ampicillin is the preferred antibiotic used to treat ampicillin-sensitive enterococcal infections. However, according to several studies comparing the therapeutic effects of beta-lactam antibiotics and vancomycin on ampicillin-susceptible enterococcal BSI, there was no significant difference in mortality [24]. Therefore, when needed, or when beta-lactam antibiotics are difficult to use, vancomycin can be considered as a treatment option for ampicillin-susceptible strains.

An important concern when using vancomycin is the occurrence of acute kidney injury. A higher vancomycin trough concentration increases the risk of VIN [20, 25]. Although most vancomycin-induced nephrotoxic events are reversible, many studies support there is increased mortality from AKI of any cause, including vancomycininduced nephropathy [26]. While 6 of our 37 patients developed VIN, it was not statistically related to trough concentration $(p=0.09)$ or 28 -day mortality. $(p=0.446)$.

In this study, mortality was higher at younger ages. Aging process causes structural and functional changes in multiple organ systems, especially the kidneys, and is known to affect PK/PD of drugs by causing changes in body composition, drug absorption, distribution, and clearance [27]. These changes may have affected the patient's prognosis or treatment outcome.

This study has several limitations that are inherent to its retrospective design. As with any observational study, there remains a possibility that unmeasured confounders influenced our findings. According to several studies, it is well known that sepsis or septic shock affects drug absorption, distribution and other pharmacological processes due to pathophysiological changes such as decreased perfusion to body organs, interstitial edema or increased capillary permeability [28]. Therefore, the PK/ PD alterations may not have been reflected depending on the patients' disease severity. In addition, in the early stage of sepsis, extravascular volume expansion with fluid loading and capillary leak causes a change in volume distribution, which can cause hyperfiltration in the kidney and lead to an alteration of drug concentration. Thereby, it may have affected the prognosis of non-survivors with low trough levels. For these reasons, we cannot exclude the possibility that residual bias affected our findings. Another limitation of this study was that only patients with bacteremia were enrolled, and patients with nonbacteremic sepsis were not analyzed. In addition, the single-center nature of a referral tertiary hospital leads to selection bias in severe cases admitted to our hospital. A multicenter study with a larger sample size is needed.

\section{Conclusions}

In conclusion, a vancomycin trough level of $15 \mu \mathrm{g} / \mathrm{mL}$ or lower was significantly associated with 28-day mortality in patients with enterococcal bacteremia. However, larger prospective studies are needed to examine the association of vancomycin PK/PD parameters for treating other gram-positive pathogens, especially Enterococcus species.

\section{Abbreviations}

AUC: Area under the concentration-time curve; MIC: Minimal inhibitory concentration; PK: Pharmacokinetics; PD: Pharmacodynamics; TDM: Therapeutic drug monitoring; VIN: Vancomycin-induced nephropathy. 


\section{Supplementary Information}

The online version contains supplementary material available at https://doi. org/10.1186/s12879-021-06809-x.

Additional file 1: Table S1. Baseline characteristics and clinical features according to Enterococcus species in patients with enterococcal bacteremia.

\section{Acknowledgements}

Not applicable.

\section{Authors' contributions}

Conceptualization:NSK, S-GL. Data collection:YS. Data analysis and interpretation: YS, JHR, NSK,S-GL. Supervision: YC, JH, YB, MK, JHK, HS, JYA, J-BL, SJJ, JYC, J-SY,YGS. Writing-original draft: YS, JHR. Writing-review and editing: NSK, S-GL. All authors read and approved the final manuscript.

\section{Funding}

The authors received no financial support for the research, authorship, and/or publication of this article.

\section{Availability of data and materials}

All datasets generated during the current study are available from the corresponding author on reasonable request.

\section{Declarations}

\section{Ethics approval and consent to participate}

All methods were carried out in accordance with relevant guidelines and regulations under Ethics approval. The study was approved by the Institutional Review Board (IRB) of the Yonsei University Health System Clinical Trial Center (4-2020-0161). The study was retrospective and the study subjects were anonymized, the IRB waived the requirement for written informed consent from the patients.

\section{Consent for publication}

Individual cannot be identified and therefore consent for publication is not required.

\section{Competing interests}

The authors declare no potential competing interests with respect to the research, authorship, and publication of this article.

\section{Author details}

${ }^{1}$ Department of Internal Medicine, Yonsei University College of Medicine, 50-1 Yonsei-ro, Seodaemun-gu, Seoul 03722, Republic of Korea. ${ }^{2}$ AIDS Research Institute, Yonsei University College of Medicine, Seoul, South Korea. ${ }^{3}$ Department of Laboratory Medicine, Yonsei University College of Medicine, 50-1 Yonsei-ro, Seodaemun-gu, Seoul 03722, Republic of Korea.

Received: 20 June 2021 Accepted: 20 October 2021

Published online: 26 October 2021

\section{References}

1. Lebreton F, Willems RJL, Gilmore MS. Enterococcus Diversity, Origins in Nature, and Gut Colonization. In: Gilmore MS, Clewell DB, Ike Y, Shankar N, editors. Enterococci: From Commensals to Leading Causes of Drug Resistant Infection. Boston; 2014.

2. Ryu BH, Hong J, Jung J, Kim MJ, Sung H, Kim MN, Chong YP, Kim SH, Lee SO, Kim YS, et al. Clinical characteristics and treatment outcomes of Enterococcus durans bacteremia: a 20-year experience in a tertiary care hospital. Eur J Clin Microbiol Infect Dis. 2019;38(9):1743-51.

3. Weiner LM, Webb AK, Limbago B, Dudeck MA, Patel J, Kallen AJ, Edwards JR, Sievert DM. Antimicrobial-Resistant Pathogens Associated With Healthcare-Associated Infections: Summary of Data Reported to the National Healthcare Safety Network at the Centers for Disease
Control and Prevention, 2011-2014. Infect Control Hosp Epidemiol. 2016;37(11):1288-301.

4. Garcia-Solache M, Rice LB. The Enterococcus: a Model of Adaptability to Its Environment. Clin Microbiol Rev. 2019;32:2.

5. Clark L, Skrupky LP, Servais R, Brummitt CF, Dilworth TJ. Examining the Relationship Between Vancomycin Area Under the Concentration Time Curve and Serum Trough Levels in Adults With Presumed or Documented Staphylococcal Infections. Ther Drug Monit. 2019;41(4):483-8.

6. Rybak M, Lomaestro B, Rotschafer JC, Moellering R, Jr., Craig W, Billeter M, Dalovisio JR, Levine DP. Therapeutic monitoring of vancomycin in adult patients: a consensus review of the American Society of HealthSystem Pharmacists, the Infectious Diseases Society of America, and the Society of Infectious Diseases Pharmacists. Am J Health Syst Pharm. 2009:66(1):82-98

7. Holmes NE, Turnidge JD, Munckhof WJ, Robinson JO, Korman TM, O'Sullivan MV, Anderson TL, Roberts SA, Warren SJ, Gao W, et al. Vancomycin AUC/MIC ratio and 30-day mortality in patients with Staphylococcus aureus bacteremia. Antimicrob Agents Chemother. 2013;57(4):1654-63.

8. Hale CM, Seabury RW, Steele JM, Darko W, Miller CD. Are Vancomycin Trough Concentrations of 15 to $20 \mathrm{mg} / \mathrm{L}$ Associated With Increased Attainment of an AUC/MIC $>/=400$ in Patients With Presumed MRSA Infection? J Pharm Pract. 2017;30(3):329-35.

9. Makmor-Bakry M, Ahmat A, Shamsuddin A, Lau CL, Ramli R. Association between single trough-based area under the curve estimation of vancomycin and treatment outcome among methicillin-resistant Staphylococcus aureus bacteremia patients. Anaesthesiol Intensive Ther. 2019:51(3):218-23.

10. Rybak MJ, Le J, Lodise TP, Levine DP, Bradley JS, Liu C, Mueller BA, Pai MP, Wong-Beringer $\mathrm{A}$, Rotschafer JC, et al. Therapeutic monitoring of vancomycin for serious methicillin-resistant Staphylococcus aureus infections: A revised consensus guideline and review by the American Society of Health-System Pharmacists, the Infectious Diseases Society of America, the Pediatric Infectious Diseases Society, and the Society of Infectious Diseases Pharmacists. Am J Health Syst Pharm. 2020;77(11):835-64.

11. Neely MN, Kato L, Youn G, Kraler L, Bayard D, van Guilder M, Schumitzky A, Yamada W, Jones B, Minejima E. Prospective trial on the use of trough concentration versus area under the curve to determine therapeutic vancomycin dosing. Antimicrob Agents Chemother. 2018;62:2.

12. Neely MN, Youn G, Jones B, Jelliffe RW, Drusano GL, Rodvold KA, Lodise TP. Are vancomycin trough concentrations adequate for optimal dosing? Antimicrob Agents Chemother. 2014;58(1):309-16.

13. Horan TC, Andrus M, Dudeck MA. CDC/NHSN surveillance definition of health care-associated infection and criteria for specific types of infections in the acute care setting. Am J Infect Control. 2008;36(5):309-32.

14. Clinical and laboratory standards institute. Performance standards for antimicrobial susceptibility testing. Clinical and laboratory standards institute; 2019. https://www.google.com/url?sa $=t \& r c t=j \& q=\& e s r c=s \&$ source $=$ web\&cd $=\&$ ved $=2$ ahUKEwiSw6zK3urqAhVX62EKHTOIATMQFj ABegQIARAB\&url=https\%3A\%2F\%2Fclsi.org\%2Fmedia\%2F2663\%2Fm10 0ed29_sample.pdf\&usg=AOvVaw0bK-ZFDfbqRfzJz03o_pWy. Accessed 26 July 2020.

15. Holmes NE, Tong SY, Davis JS, van Hal SJ. Treatment of methicillin-resistant Staphylococcus aureus: vancomycin and beyond. Semin Respir Crit Care Med. 2015;36(1):17-30.

16. Rybak MJ. The pharmacokinetic and pharmacodynamic properties of vancomycin. Clin Infect Dis. 2006;42 Suppl 1:S35-9.

17. Alvarez R, Lopez Cortes LE, Molina J, Cisneros JM, Pachon J. Optimizing the Clinical Use of Vancomycin. Antimicrob Agents Chemother. 2016;60(5):2601-9.

18. Mogle BT, Steele JM, Seabury RW, Dang UJ, Kufel WD. Implementation of a two-point pharmacokinetic AUC-based vancomycin therapeutic drug monitoring approach in patients with methicillin-resistant Staphylococcus aureus bacteraemia. Int J Antimicrob Agents. 2018;52(6):805-10.

19. Jumah MTB, Vasoo S, Menon SR, De PP, Neely M, Teng CB. Pharmacokinetic/Pharmacodynamic Determinants of Vancomycin Efficacy in Enterococcal Bacteremia. Antimicrob Agents Chemother. 2018:62:3.

20. Nakakura I, Sakakura K, Imanishi K, Sako R, Yamazaki K. Association between vancomycin pharmacokinetic/pharmacodynamic parameters, patient characteristics, and mortality in patients with bacteremia caused by vancomycin-susceptible Enterococcus faecium: a single-center retrospective study. J Pharm Health Care Sci. 2019;5:8. 
21. Zelenitsky S, Rubinstein E, Ariano R, lacovides H, Dodek P, Mirzanejad Y, Kumar A, Cooperative Antimicrobial Therapy of Septic Shock CDRG. Vancomycin pharmacodynamics and survival in patients with methicillinresistant Staphylococcus aureus-associated septic shock. Int J Antimicrob Agents. 2013;41(3):255-60.

22. Kullar R, Davis SL, Taylor TN, Kaye KS, Rybak MJ. Effects of targeting higher vancomycin trough levels on clinical outcomes and costs in a matched patient cohort. Pharmacotherapy. 2012;32(3):195-201.

23. Meng L, Fang Y, Chen Y, Zhu H, Long R. High versus low vancomycin serum trough regimen for Gram-positive infections: a meta-analysis. J Chemother. 2015;27(4):213-20.

24. Petersiel N, Bitterman R, Manaa A, Nashashibi L, Moskovich O, Geffen Y, Polak D, Braun E, Neuberger A, Paul M. beta-lactam antibiotics vs. vancomycin for the early treatment of enterococcal bacteraemia: A retrospective cohort study. Int J Antimicrob Agents. 2019;53(6):761-6.
25. Hammoud K, Brimacombe M, Yu A, Goodloe N, Haidar W, El Atrouni W. Vancomycin Trough and Acute Kidney Injury: A Large Retrospective, Cohort Study. Am J Nephrol. 2016;44(6):456-61.

26. Bamgbola O. Review of vancomycin-induced renal toxicity: an update. Ther Adv Endocrinol Metab. 2016;7(3):136-47.

27. Shi S, Klotz U. Age-related changes in pharmacokinetics. Curr Drug Metab. 2011;12(7):601-10.

28. Charlton M, Thompson JP. Pharmacokinetics in sepsis. BJA Educ. 2019;19(1):7-13.

\section{Publisher's Note}

Springer Nature remains neutral with regard to jurisdictional claims in published maps and institutional affiliations.
Ready to submit your research? Choose BMC and benefit from:

- fast, convenient online submission

- thorough peer review by experienced researchers in your field

- rapid publication on acceptance

- support for research data, including large and complex data types

- gold Open Access which fosters wider collaboration and increased citations

- maximum visibility for your research: over $100 \mathrm{M}$ website views per year

At BMC, research is always in progress.

Learn more biomedcentral.com/submissions 